\title{
Semaphorin-5B Controls Spiral Ganglion Neuron Branch Refinement during Development
}

\author{
Johnny S. Jung, ${ }^{1}$ Kaidi D. Zhang, ${ }^{1}$ Zhirong Wang, ${ }^{1}$ Mark McMurray, ${ }^{2}$ Andrew Tkaczuk, ${ }^{2}$ Yoko Ogawa, ${ }^{2}$ \\ Ronna Hertzano, ${ }^{2,3,4}$ and ${ }^{-T h o m a s ~ M . ~ C o a t e ~}{ }^{1}$ \\ ${ }^{1}$ Department of Biology, Georgetown University, Washington, DC 20007, and Departments of ${ }^{2}$ Otorhinolaryngology Head and Neck Surgery, ${ }^{3}$ Anatomy and \\ Neurobiology, and ${ }^{4}$ Institute for Genome Sciences, University of Maryland School of Medicine, University of Maryland, Baltimore, Maryland 21201
}

During nervous system development, axons often undergo elaborate changes in branching patterns before circuits have achieved their mature patterns of innervation. In the auditory system, type I spiral ganglion neurons (SGNs) project their peripheral axons into the cochlear epithelium and then undergo a process of branch refinement before forming synapses with sensory hair cells. Here, we report that Semaphorin-5B (Sema5B) acts as an important mediator of this process. During cochlear development in mouse, immature hair cells express Sema5B, whereas the SGNs express both PlexinA1 and PlexinA3, which are known Sema5B receptors. In these studies, genetic sparse labeling and three-dimensional reconstruction techniques were leveraged to determine the morphologies of individual type I SGNs after manipulations of Sema5B signaling. Treating cultured mouse cochleae with Sema5B-Fc (to activate Plexin-As) led to type I SGNs with less numerous, but longer terminal branches. Conversely, cochleae from Sema5b knock-out mice showed type I SGNs with more numerous, but shorter terminal branches. In addition, conditional loss of Plxnal in SGNs (using Bhlhb5 ${ }^{\mathrm{Cre}}$ ) led to increased type I SGN branching, suggesting that PlexinA1 normally responds to Sema5B in this process. In these studies, mice of either sex were used. The data presented here suggest that Sema5B-PlexinA1 signaling limits SGN terminal branch numbers without causing axonal repulsion, which is a role that distinguishes Sema5B from other Semaphorins in cochlear development.

Key words: auditory; axon guidance; cochlea; Plexin; semaphorin; spiral ganglion

\section{Significance Statement}

The sensorineural components of the cochlea include hair cells, which respond mechanically to sound waves, and afferent spiral ganglion neurons (SGNs), which respond to glutamate released by hair cells and transmit auditory information into the CNS. An important component of synapse formation in the cochlea is a process of SGN "debranching" whereby SGNs lose extraneous branches before developing unramified bouton endings that contact the hair cells. In this work, we have found that the transmembrane ligand Semaphorin-5B and its receptor PlexinA1 regulate the debranching process. The results in this report provide new knowledge regarding the molecular control of cochlear afferent innervation.

\section{Introduction}

A wide diversity of neurons constitutes the vertebrate nervous system, each type characterized by a morphology that is condu-

Received Jan. 14, 2019; revised May 3, 2019; accepted June 10, 2019.

Author contributions: J.S.J., K.D.Z., and T.M.C. designed research; J.S.J., K.D.Z., Z.W., M.M., A.T., Y.O., and R.H. performed research; J.S.J., K.D.Z., and M.M. analyzed data; J.S.J. and T.M.C. wrote the paper.

This work was supported by NIH ROO DC13107 (T.M.C.) and NIH R01, DC013817 (R.H.). We thank the members of the Coate and Hertzano laboratories for their valuable discussions, and Dr. Matthew Kelley (National Institute on Deafness and Other Communication Disorders) and Paige Brooks (Georgetown University) for critical reading of the paper. The Sema5b-null line was a kind gift from Dr. Alex Kolodkin (Johns Hopkins University), the Neurog ${ }^{\text {CreeRT2 line was }}$ a kind gift from Dr. Lisa Goodrich (Harvard University), the Bhlhb5 ${ }^{\text {Cre }}$ was used with permission from Dr. Michael Greenberg (Harvard University), and the Plxna ${ }^{\text {f }}$ line was a kind gift from Dr. Yutaka Yoshida (Cincinnati Children's Hospital).

The authors declare no competing financial interests.

Correspondence should be addressed to Thomas M. Coate at tmc91@georgetown.edu.

https://doi.org/10.1523/JNEUROSCI.0113-19.2019

Copyright $\odot 2019$ the authors cive to its specific function. During development, neurons pair with their synaptic targets and simultaneously undergo (sometimes elaborate) changes in branching morphology (Kalil and Dent, 2014; Riccomagno and Kolodkin, 2015; Schuldiner and Yaron, 2015). In certain cases, as with thalamocortical axons, neuronal branching and arborization become increasingly complex over developmental time (Agmon et al., 1993). By contrast, neurons like L5 cortical neurons, undergo dramatic periods of pruning, fragmentation, and refinement (Stanfield et al., 1982). Extracellular cues that modulate axonal branch patterns act through Rho GTPases and other cytosolic factors to regulate changes in cytoskeletal dynamics (Spillane and Gallo, 2014). Given that axon branching can affect the functional properties of a neuron (Yoong et al., 2019), it is important to consider how cues in development dictate the differences in branch morphol- 
ogy. In this report, we show that Semaphorin-5B controls the branching behaviors of auditory neurons within the cochlea.

In the cochlea, hair cells are organized into one row of inner hair cells (IHCs), and three rows of outer hair cells (OHCs). Hair cells are mechanoreceptors responsible for detecting sound waves and releasing glutamate onto spiral ganglion neurons (SGNs; Glowatzki and Fuchs, 2002). IHCs are innervated by type I SGNs, which represent $\sim 95 \%$ of the total SGN population. By contrast, type II SGNs ( $\sim 5 \%$ of the population) pass by the IHCs and innervate 15 or more OHCs (Berglund and Ryugo, 1987; Raphael and Altschuler, 2003). IHCs and type I SGNs mediate our primary sense of hearing, whereas type II SGNs are now thought to mediate auditory nociception (Flores et al., 2015; Liu et al., 2015). To achieve this stereotyped innervation pattern in the cochlea, the SGNs undergo a highly dynamic period of extension, retraction, and refinement (Druckenbrod and Goodrich, 2015). At E15.5 in mice, peripheral processes of SGNs are highly branched and exploratory (Koundakjian et al., 2007), with approximately one-half of all SGNs extending into the $\mathrm{OHC}$ region (Coate et al., 2015). By early postnatal stages, nearly all of the type I SGNs have receded to the IHC region as a result of Ephrin-A5 (Defourny et al., 2013) and Sema3F (Coate et al., 2015) repulsive cues. Previously, SGN fate-mapping experiments revealed that type I SGNs undergo a debranching process around the time of inner hair cell targeting, and that the loss of extraneous terminal branches coincides with SGN maturation (Koundakjian et al., 2007).

First recognized as axon guidance cues in the nervous system, the Semaphorins (Semas) are also known to regulate events such as synaptogenesis, dendritic spine formation, and neural plasticity (de Wit and Verhaagen, 2003; Fiore and Püschel, 2003; Pasterkamp and Giger, 2009). Vertebrate Semas are extracellular cues that are either secreted (Sema3s) or membrane-bound (Sema4s-Sema7s) and bind Neuropilin (Nrp) and/or Plexin receptors to modulate cytoskeletal dynamics (Jongbloets and Pasterkamp, 2014). The effects of Sema3s have been shown previously in inner ear development: Sema3A/Nrp1 signaling controls vestibular sensory targeting (Gu et al., 2003; Katayama et al., 2013) and SGN survival (Salehi et al., 2017), and Sema3F/Nrp2 signaling is required for type I SGN retraction (Coate et al., 2015). However, nothing is known about the functional roles of transmembrane Semas in inner ear development, and here we have focused our attention on Sema5B. Most of the current understanding of Sema5B comes from work in the mouse retina and chick spinal cord where Sema5B acts as a repulsive cue by signaling through PlexinA1 and PlexinA3 (Matsuoka et al., 2011; Liu et al., 2014). Sema5B has also been shown to eliminate synaptic connections in hippocampal neurons in vitro (O'Connor et al., 2009). Here, our findings suggest that Sema5B is expressed in developing cochlear hair cells and signals to PlexinA1 on SGNs. Rather than acting as a traditional repulsive cue, Sema5B signaling appears to limit type I SGN branch numbers as part of SGN refinement.

\section{Materials and Methods}

Mouse lines. All mice were maintained in accordance with the Animal Care and Use Committees at Georgetown University and the University of Maryland School of Medicine. For the Sema5b in situ hybridization studies, timed-pregnant ICR mice (Charles River Laboratories) were used. Timed-pregnant NIH Swiss mice (Charles River Laboratories) were used for the Plxn in situ hybridization studies, and the protein expression studies shown in Figure 2. Timed-pregnant mice carrying the Neurog1 ${ }^{\text {CreERT2 }}$ transgene (Koundakjian et al., 2007) were crossed with mice carrying the Rosa26 ${ }^{\text {tdTomato }}$ reporter allele (Jackson Laboratories, stock\# 007914) to generate mice for labeling sparse numbers of SGNs. These alleles will be sometimes abbreviated " $N^{\text {CreERT2 }} ; R^{\text {Tom }}$." Previously, we and others found that embryos carrying these alleles show beautiful labeling of individual SGNs without the addition of tamoxifen (Coate et al., 2015; Druckenbrod and Goodrich, 2015). $N^{\text {CreERT2 }} ; R^{\text {Tom }}$ mice were used for cochlear culture experiments and crossed to Sema5bnull mice. The Sema5b-null mice (Matsuoka et al., 2011) were maintained on a C57BL6 background until interbred with the $N^{\text {CreERT2 }} ; R^{\text {Tom }}$ mice, which were of mixed background strain. Bhlhb5 ${ }^{\text {Cre }}$ (Ross et al., 2010) and Plxnal ${ }^{\mathrm{fl}}$ (Yoshida et al., 2006) mice were maintained on a C57BL6 background and cross-bred for selective removal of Plxna1 in SGNs (efficacy of Bhlhb5 ${ }^{\text {Cre }}$ in SGNs shown by Ghimire et al. (2018). Gfil ${ }^{\text {Cre }}$ mice (Yang et al., 2010) were maintained on a C57BL6 background and used to remove Plxnal in hair cells; this experiment was performed to examine the specificity of the PlexinAl antibody staining in the hair cell stereocilia (Fig. 2). In all experiments, mice of either sex were used.

In vitro culture of embryonic cochleae. For SGN cultures, E16.5-P1 cochleae from $N^{\text {CreERT2 }} ; R^{\text {Tom }}$ mice were isolated and roofs of the cochlear ducts were carefully removed. The cochleae were then cultured for $24 \mathrm{~h}$ at $37^{\circ} \mathrm{C}$ at $5 \% \mathrm{CO}_{2}$ on polycarbonate membrane filters (Sterlitech PCT0213100) in media described previously (Coate et al., 2015). Sema5B-Fc chimera protein (R\&D Systems, 6680-S5-050) or control purified human IgG-Fc (Jackson ImmunoResearch, 009-000-008) were added to the medium at a final concentration of $23 \mathrm{~nm}$. Typically, to induce cellular events such as growth cone collapse, Sema-Fc proteins are used in concentrations ranging from 1 to $100 \mathrm{~nm}$ (Oster et al., 2003; Salehi et al., 2017). Following the culture period, the cochleae were collected and fixed in $4 \%$ paraformaldehyde for $10 \mathrm{~min}$, then processed for immunostaining and confocal microscopy.

In situ hybridization. Mouse heads with brains removed were fixed in $4 \%$ paraformaldehyde overnight and rinsed in $1 \times$ PBS. Isolated inner ears were treated with 10,20 , and $30 \%$ sucrose before being embedded in OCT Compound (Tissue-Tek). The frozen blocks were sectioned at 12 $\mu \mathrm{m}$ and in situ hybridization was performed essentially as described previously (Driver et al., 2008). The Sema 5 b cDNA fragment was cloned using forward (GGCCTGCCCAGAAGGCTGGTCACTG) and reverse (CACTGACAAGCTGGACGCAGCCCCG) primers and was built into the pCRII-TOPO plasmid. Plxna1- and Plxna3-containing plasmids for in situ hybridization (Schwarz et al., 2008; Bouvrée et al., 2012) were kind gifts from Christiana Ruhrberg (University College London Institute of Ophthalmology) and are also available at Addgene (catalog \#s58237 and \#58238). In situ probes used in this study and how they were generated: Sema $5 b$ antisense: Spe1 digestion, T7 transcription; Sema $5 b$ sense: Xho1 digestion, Sp6 transcription; Plxna1 antisense: EcoR1 transcription,T7 polymerase; Plxna1 sense: Not1 transcription, T3 polymerase; Plxna3 antisense: Xho1 transcription, T7 polymerase; Plxna3 sense: EcoRV digestion, $\mathrm{T} 3$ transcription.

Immunostaining. Whole heads or isolated inner ears were fixed with $4 \%$ paraformaldehyde for $45 \mathrm{~min}$ then rinsed in $1 \times$ PBS. For section immunostaining, samples were prepared and sectioned as described in the section on in situ hybridization. For whole-mount immunostaining of cochleae, the cochlear capsules and stria were removed, then the samples were permeabilized in PBS supplemented with $0.5 \%$ Triton X-100, then blocked in $10 \%$ normal horse serum (Vector Laboratories, S-2000) for at least $1 \mathrm{~h}$. The immunostaining procedure was performed as previously described (Coate et al., 2015). Antibodies used in this study include the following (Table 1): mouse-anti-Tuj1 (Covance, MMS-435P; 1:500), rabbit-anti-GAP43 (Millipore, AB5220; 1:1000), rabbit-anti-dsRed (Clontech, 632496; 1:2000), rabbit-anti-MyosinVI (MyoVI; Proteus Biosciences, 25-6791; 1:1000), goat-anti-Sox2 (R\&D Systems, AF2018; 1:400), goat-antiMyoVI (Coate et al., 2015), goat-anti-PlexinA1 (R\&D Systems, AF4309; 1:100), goat-anti-PlexinA3 (R\&D Systems, AF4075; 1:100), and chickenanti-neurofilament (NF200; Aves Labs, NFH; 1:2000).

Auditory brainstem response. Sema $5 b^{-1-}$ and littermate control mice at P40 were anesthetized and analyzed for auditory brainstem response (ABR) thresholds. The ABR procedure was previously described (Matern et al., 2017). Sound stimuli were $5 \mathrm{~ms}$ tone bursts at four different fre- 
Table 1. Primary antibodies and tools/software used in this study

\begin{tabular}{|c|c|c|c|}
\hline Product & Category & Source/vendor (catalog \#) & RRID \\
\hline Mouse $\alpha$-Tuj1 (TUBB3) & Antibody & BioLegend (801213) & $A B \_2728521$ \\
\hline Rabbit $\alpha$-Gap43 & Antibody & Millipore (AB5220) & AB_2107282 \\
\hline Rabbit $\alpha$-dsRed & Antibody & Takara Bio (632496) & $A B \_10013483$ \\
\hline Rabbit $\alpha$-Myo6 & Antibody & Proteus Biosciences (25-6791) & $A B \_10013626$ \\
\hline Goat $\alpha$-Sox2 & Antibody & R\&D Systems (AF2018) & $A B \_355110$ \\
\hline Goat $\alpha$-Myo6 & Antibody & Section on Developmental Neuroscience; NIDCD & $A B \_2783873$ \\
\hline Goat $\alpha$-PIxnA1 & Antibody & R\&D Systems (AF4309) & $A B \_10645644$ \\
\hline Goat $\alpha$-PIxnA3 & Antibody & R\&D Systems (AF4075) & $A B \_2166395$ \\
\hline Chicken $\alpha$-NF200 & Antibody & Aves Labs (NFH) & $A B \_2313552$ \\
\hline Human lgG-Fc protein & Antibody & Jackson ImmunoResearch Labs (009-000-008) & $A B \_2337046$ \\
\hline Bitplane Imaris & Tools/Software & 0xford Instruments & SCR_007370 \\
\hline GraphPad Prism & Tools/Software & GraphPad & SCR_002798 \\
\hline Fiji & Tools/Software & ImageJ & $S C R \_002285$ \\
\hline
\end{tabular}

quencies $(8,16,24,32 \mathrm{kHz}$ ) from 90 to $0 \mathrm{~dB}$ (with $5 \mathrm{~dB}$ decrement each time). Hearing thresholds were determined by looking at average ABR waveforms from 512 recordings per condition.

Imaging. Confocal $z$-stack images of immunohistochemistry samples were taken on a Zeiss LSM-880 confocal. Micrographs of in situ hybridization samples were taken using a Nikon E800 compound scope and Nikon DXM1200 digital camera.

Experimental design and statistical analyses. For quantification of individual SGN morphologies, anti-dsRed immunostaining was performed and confocal $z$-stack images of SGN peripheral processes were acquired. From $z$-stack images, dsRed-positive SGNs in $N^{\text {CreERT2 }} ; R^{\text {Tom }}$ mice were traced and reconstructed using the filaments feature in Imaris (Bitplane), from which various parameters of SGN morphology were calculated and are defined as follows (see Fig. 3E). Branch number: the number of segments present between all neuronal branching points or terminal points in a single peripheral process. Average branch depth: each branch (as defined previously) is assigned a branch depth value, which increases relative to the number of branching points between it and the main axon of the peripheral process. The average branch depth is the mean of all branch depth values in a single peripheral process. Average branch length: the mean length, in micrometers, of all branches (excluding the main axon shaft beneath the terminal arborization) in a single peripheral process. Total branch length: the sum of lengths, in micrometers, of all branches (excluding the main axon shaft beneath the terminal arborization) in a single peripheral process. Statistical analyses were performed using Prism (GraphPad). For occupied area analyses, $z$-stack images were processed into max intensity projections and ROIs of fixed heights (18 $\mu \mathrm{m}$ ) were drawn centered on the modiolar face of IHCs in Fiji (Schindelin et al., 2012), as shown in Figure 6, H and $I$. Immunofluorescent NF200 images were converted into binary signal images using the mean autothreshold binarization function, as described previously (Glasbey, 1993). The area within ROIs occupied by signal was calculated using the Analyze Particles function and reported as a percentage of the initial ROI area. See figure legends for statistical tests and sample sizes.

\section{Results}

Differentiating hair cells express Sema5b, whereas SGNs express Plxna1 and Plxna3

The cochlear sensory domain is comprised of a single row of IHCs and three rows of OHCs, which are innervated by type I and II SGNs respectively. Morphologically, the SGNs are bipolar: they extend peripheral axons (sometimes termed "dendrites") that innervate hair cells, and central axons that project out of the inner ear and innervate neurons within the cochlear nucleus (Nayagam et al., 2011; Shepard et al., 2019). Figure $1 A-C$ shows wholemount or cross-sectional views of these structures with the SGNs labeled with anti-NF200 antibodies and the hair cells labeled with anti-MyoVI antibodies. Before the formation of synapses and subsequent transmission of auditory input, the SGNs go through an elaborate phase of targeting and branch refinement that coin- cides with hair cell differentiation (Fig. 1D). At $\sim$ E15.5, the SGNs send highly branched peripheral axons into both the IHC and OHC regions (Koundakjian et al., 2007; Coate et al., 2015). At $\sim$ E16.5, the type I SGNs retract from the OHC region to the IHC region (Coate et al., 2015; Druckenbrod and Goodrich, 2015) and the type II SGNs turn toward the base. Throughout this process, the type I SGNs shed their terminal branches as a prelude to establishing single, unramified bouton endings with IHCs (Coate et al., 2019). In the mature mouse cochlea, a single IHC will accommodate as many as 20 SGN synaptic endings (Meyer and Moser, 2010). During development, transient immature synapses are likely formed and eliminated, with permanent synapses being established around the end of the first postnatal week (Huang et al., 2012), and hearing commencing as early as P10 in mice (Polley et al., 2013).

Several online databases showed that $S e m a 5 b$ was expressed in the developing cochlear sensory domain. These included the gEAR database (https://umgear.org/), the Allen Brain Atlas (Allen Institute for Brain Science, 2015; http://developingmouse. brain-map.org/gene/show/20120), and Embryo Express (http:// www.embryoexpress.org/). Our own in situ hybridization experiments show that Sema5b is expressed by hair cells as early as E16.5 (Fig. 1E), maintained in hair cells through P1 (Fig. $1 F$ ), and absent from hair cells by P5 (Fig. $1 G$ ). These data show that Sema5b/Sema5B is expressed by hair cells during early phases of hair cell innervation when SGNs undergo selective hair cell targeting and terminal branch refinement. In previous work in the retina, it was shown that PlexinA1 and PlexinA3 are possible receptors for Sema5B (Matsuoka et al., 2011). Therefore, we asked whether PlexinA1 and PlexinA3 expression in the cochlea showed temporal overlap with Sema5B. We note here that PlexinA1 and PlexinA3 were previously shown to be expressed by SGNs at E16.5 (Murakami et al., 2001; Coate et al., 2015), but other stages were not examined. To examine Plxna1 and -a3 mRNA distribution, we performed in situ hybridization on cochlear cross sections at E17.5 using antisense DIG-labeled probes (Fig. $1 H-K$ ). Transcripts for both receptors were clearly evident in the SGNs at this stage ( $H$ and $J$ ). In addition, Plxnal, but not Plxna3 transcripts were apparent in the lateral (abneural) region of the cochlear sensory domain (Fig. $1 H$, arrowhead). Plxnal and Plxna3 sense control probes showed no reactivity demonstrating the antisense probes are specific (Fig. $1 I, K$ ).

\section{PlexinA1 and PlexinA3 protein expression during cochlear development}

We next examined the distribution of PlexinA1 and PlexinA3 proteins by immunostaining cross-sections of cochleae from dif- 

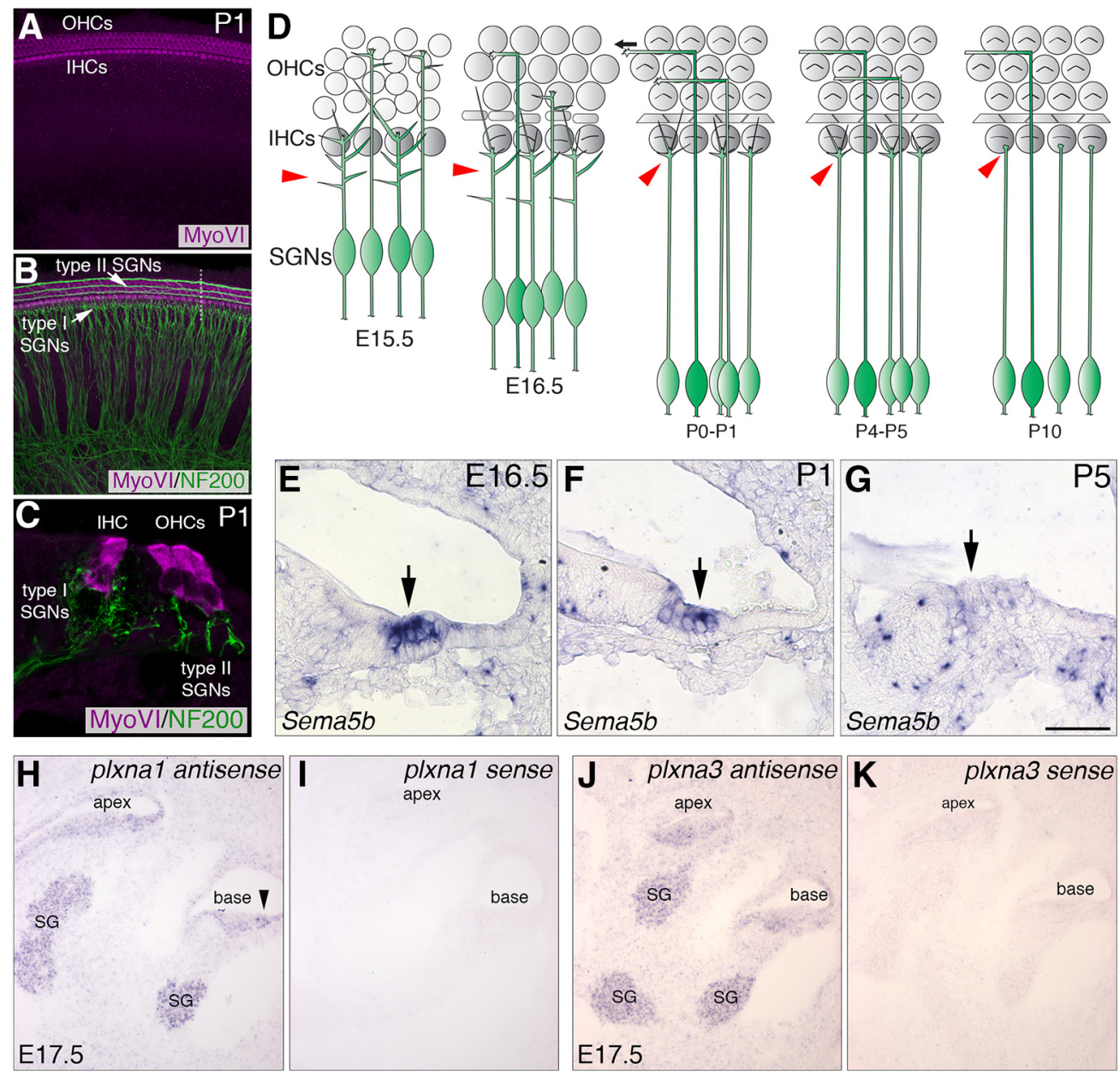

Figure 1. SGNs undergo a stereotyped refinement processes while Sema $5 b$ and $P$ Ixnas are expressed in differentiating hair cells and SGNs, respectively. $A$, A whole-mount view of hair cells from a P1 cochlea, shown by MyoVI labeling, organized into one row of inner hair cells (IHCS) and three rows of outer hair cells (OHCS). $\boldsymbol{B}$, Sample from $\boldsymbol{A}$ with NF200 labeling of type I and II SGNs. C, A cross-sectional view of the same structures and labeling from the orientation of the dotted line in $\boldsymbol{B}$. D. At E15.5, immature SGN peripheral axons have many terminal branches (red arrowheads), which explore the differentiating cochlear sensory domain. A few days before birth, SGNs begin to retract and remove excess branches, as type-ISGNs prepare to ultimately form a single connection with a single IHC. $\boldsymbol{E}-\boldsymbol{G}$, In situ hybridizations showing that Sema5b mRNA is specifically expressed in hair cells, identified by arrows, in late embryonic $(\boldsymbol{E})$ and early postnatal $(\boldsymbol{F})$ development, but is largely eliminated by P5 (G). $\boldsymbol{H}-\boldsymbol{K}, P / x n a$ mRNA expression in E17.5 cochlear cross-sections using Plxna1 $(\boldsymbol{H}, \boldsymbol{I})$ and Plxna3 $(\boldsymbol{J}, \boldsymbol{K})$ antisense probes along with sense controls. P/xna 1 transcripts are visible in the spiral ganglion (SG) and in lateral region of the cochlear sensory domain (arrowhead) in $\boldsymbol{H}$. Plxna3 transcripts are visible in the SG in J. Sense controls show no reactivity. Scale bar (in $\mathbf{G}$ ): $A, B, \sim 100 \mu \mathrm{m} ; C, 20 \mu \mathrm{m} ; \boldsymbol{E}-\mathbf{G}, 60 \mu \mathrm{m} ; \boldsymbol{H}-\boldsymbol{K}, 200 \mu \mathrm{m}$.

ferent time points (Fig. 2); for these samples, anti-MyoVI antibodies (blue) were used to identify hair cells, whereas anti-Tuj1 antibodies were used to mark the SGNs. At E14.5, before hair cells are visible and before the SGNs project into the cochlear epithelium, both PlexinA1 and PlexinA3 were only barely detectable over background (Fig. 2A-D). However, by E16.5 when the SGNs begin to make contact with hair cells (and MyoVI staining is visible) PlexinA1 and PlexinA 3 were evident on both the SGN cell bodies and developing peripheral axons (Fig. $2 E-H$, arrows). At P0, PlexinA1 protein levels were similar to the levels found at E16.5, or perhaps slightly higher (Fig. $2 I, J$ ). By contrast, PlexinA3 protein was clearly present on SGNs at P0, but the levels appeared to have diminished (Fig. $2 K, L$ ) relative to E16.5. In addition, PlexinAl protein was also evident on $\mathrm{OHC}$ membranes (Fig. 2M-P, arrowheads) consistent with the presence of Plxna1 mRNA in the OHC cytoplasm shown in Figure 1A. We note here that the strong PlexinA1 staining apparent on the OHC stereocilia is likely nonspecific: we detected no reduction in staining intensity in the OHC stereocilia after conditionally removing Plxna1 in OHCs using Gfil ${ }^{\mathrm{Cre}}$ and a Plxna $1^{\mathrm{fl}}$ allele (Yoshida et al.,
2006). In this experiment, PlexinA 1 was clearly removed from OHC cell membranes (Fig. 2 compare $O, P$ ), but not the OHC bundles (Fig. 2compare $O^{\prime}, P^{\prime}$ ). No hair cell developmental phenotypes were observed in the Gfi1 ${ }^{\mathrm{Cre}} ;$ Plxna $1^{\mathrm{fl} / \mathrm{fl}}$ cochleae compared with controls. Overall, the expression of PlexinA1 and PlexinA3 on SGNs is temporally and spatially complementary to the expression of Sema5B by hair cells during development. These data suggest the possibility that Sema5B/PlexinA signaling might mediate hair cell innervation by SGNs.

\section{Sema5B restricts SGN branch numbers in vitro}

Encouraged by the specific temporal and spatial expression patterns of Sema5B and its Plexin receptors, we next investigated the potential contribution of Sema5B signaling to SGN morphological development in the week before birth. First, to determine whether Sema5B can influence SGN guidance decisions, we performed a series of experiments whereby semi-intact cultured cochleae were exposed to either soluble Sema5B-Fc (the Sema5B $\mathrm{N}$-terminus conjugated to IgG-Fc) or Control-Fc. For these experiments, the high density and overlap of SGNs make it virtually 

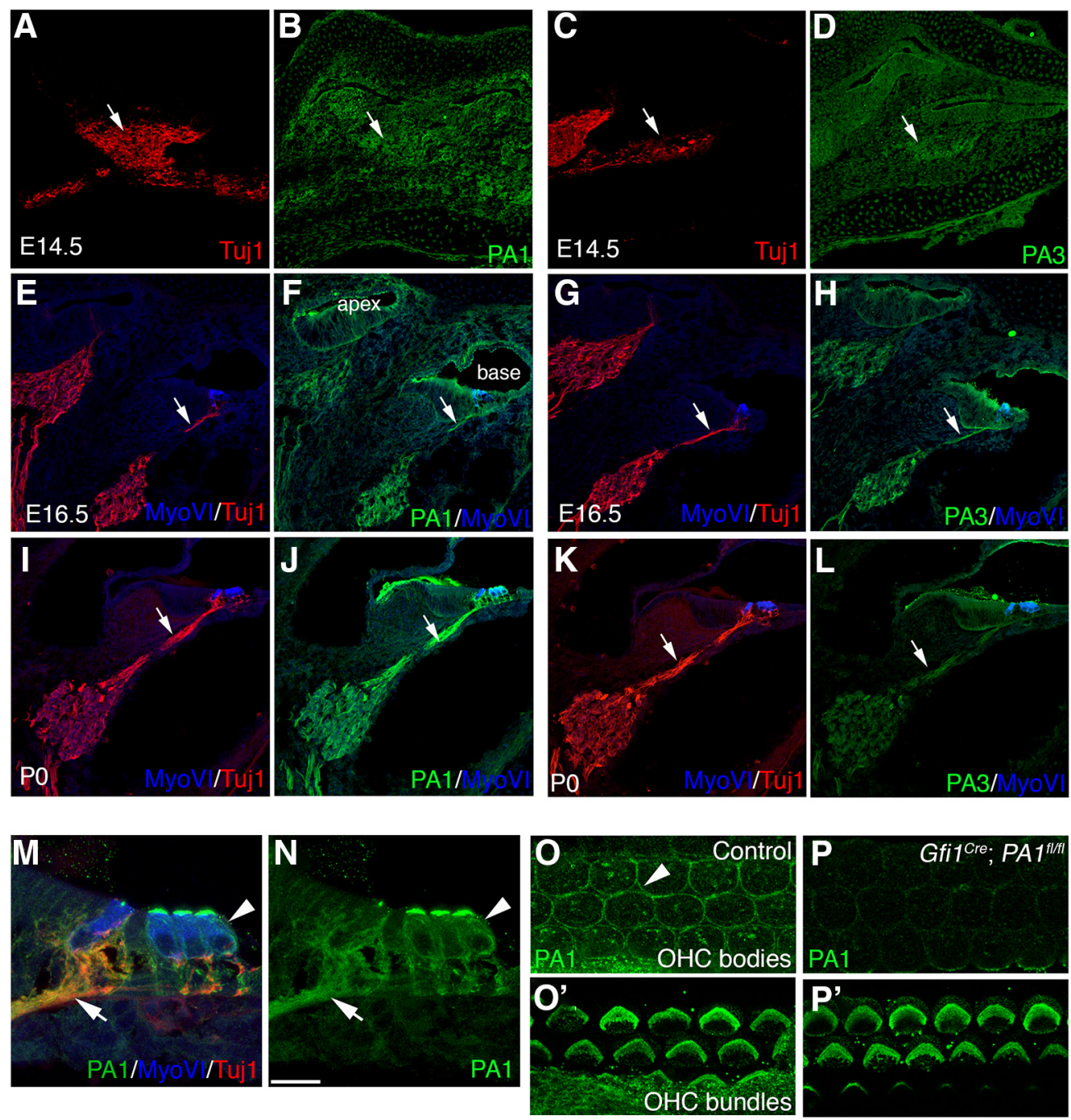

Figure 2. PlexinA1 and PlexinA3 expression in the developing cochlea. $\boldsymbol{A}-\boldsymbol{L}$, Immunostaining of WT cochlear cross-sections at E14.5 (A-D), E16.5 (E-H), and P0 (I- $\boldsymbol{L})$ with either anti-PlexinA1 or -PlexinA3. MyoVI and Tuj1 counterstaining was used where indicated. $\boldsymbol{A}-\boldsymbol{D}$, Arrows point to the SGN cell bodies. $\boldsymbol{E}-\boldsymbol{L}$, Arrows point to the SGN peripheral axons. At E14.5, both PlexinA1 (B) and PlexinA3 (D) appear only very faintly in SGNs. At E16.5, PlexinA1 $(\boldsymbol{F})$ and PlexinA3 $(\boldsymbol{H})$ proteins are clearly visible in the cell bodies and peripheral axons of SGNs; hair cells are labeled with MyoVI. Using the same staining scheme as $\boldsymbol{F}$ and $\boldsymbol{H}$ at P0, PlexinA1 is expressed at robust levels and is clearly visible in SGN peripheral axons $(\boldsymbol{J})$, as is PlexinA3, but to a lesser extent $(\boldsymbol{L})$. $\boldsymbol{M}, \boldsymbol{N}$, A

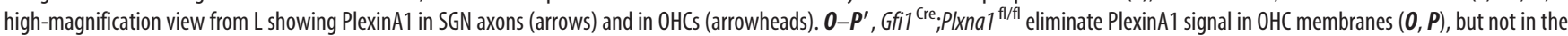
stereocilia bundles $\left(\boldsymbol{O}^{\prime}, \boldsymbol{P}^{\prime}\right)$, suggesting expression in bundles seen in $\boldsymbol{M}$ and $\boldsymbol{N}$ is likely nonspecific. $\mathbf{0}$, Arrowhead points to an $\mathbf{0 H C}$ membrane. Scale bar (in $\left.\boldsymbol{N}\right): \boldsymbol{A}-\boldsymbol{L}, \sim 100 \mu \mathrm{m} ; \boldsymbol{M}, \boldsymbol{N}, 18 \mu \mathrm{m} ; \mathbf{0}$, $P, 10 \mu \mathrm{m}$.

impossible to analyze the morphology of a single SGN in isolation. To circumvent this problem, we used cochleae from mice carrying Neurog1 ${ }^{\text {CreERT2 }}$ (Koundakjian et al., 2007) and

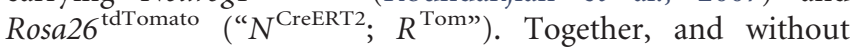
tamoxifen-mediated Cre induction, these alleles yield cochleae with sparse numbers of SGNs with bright tdTomato expression that permits neuron tracing and morphological quantification (Fig. 3A). Experiments were performed using cochleae from both E18.5 and P0 mice in correspondence to the timing of Sema5B and PlexinA1/PlexinA3 expression (Figs. 1, 2), and the period when the SGNs undergo branch refinement (Fig. 1D). After the $24 \mathrm{~h}$ culture period, the SGNs were imaged by confocal microscopy, then reconstructed and analyzed using Imaris software (Fig. $3 B, C$ ). As illustrated in Figure $3 E$, measurements of "branch depth" account for the number of secondary, tertiary, quaternary, etc., branches extending from a primary branch. Axons with a greater complexity of terminal branches have larger branch depth values, whereas axons with more limited complexity have smaller branch depth values.
Interestingly, Sema5B-Fc treatment did not cause the SGNs to retract as if they were under the influence of a strong chemorepellant, which is an effect we observed after similar experiments using Sema3A-Fc (Salehi et al., 2017). After extensive morphometric analysis and quantification, we found that Sema5B-Fc had little influence on SGN morphology when treatments were performed starting at E18.5 (Fig. $3 F-H)$ ). However, in treatments starting at P0, we found that Sema5B-Fc led to significant SGN morphological changes: Sema5B-Fc led to fewer terminal branches per SGN (Fig. $3 F$ ), and lower average branch depth values (Fig. $3 G$ ). Interestingly, whereas Sema5B treatment led to reduced terminal branch numbers and branch depth, Sema5B led to increased branch length compared with controls (Fig. $3 H$ ). The reconstructions in Figure 3D illustrate how Sema5B-treated SGNs have reduced numbers of branches, but that individual branches tend to be longer on average. Interestingly, the accumulated total of all branch lengths was not significantly different between the Sema5B-Fc treated samples and controls (Fig. 3I). This indicates that Sema5B-Fc treatment redistributed the con- 

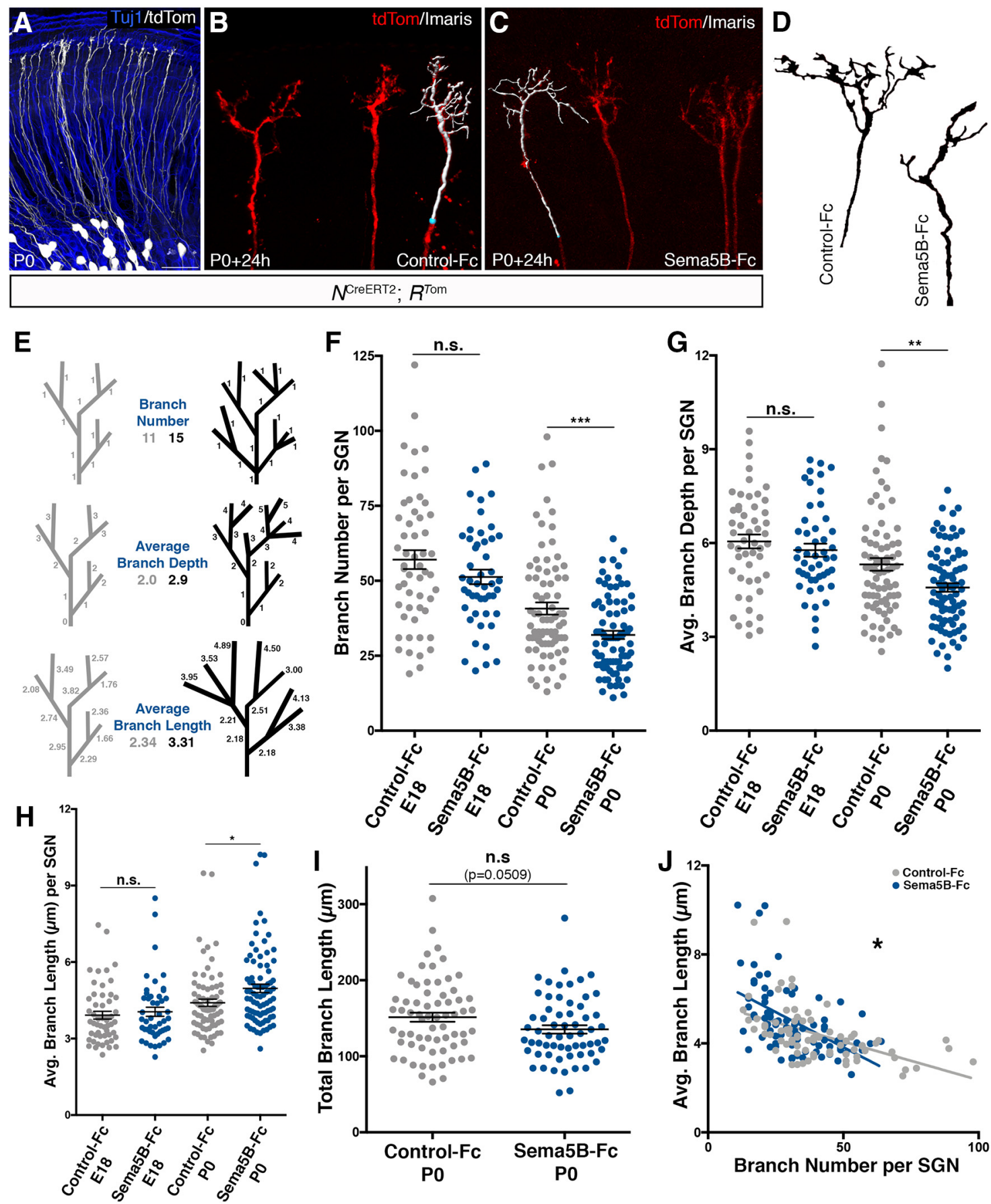

Figure 3. Sema $5 B$ limits SGN terminal branch numbers in vitro. $A$, A whole-mount view of an $N^{\text {CreeRT2 } ; ~} R^{\text {Tom }}$ cochlea showing sparse SGN labeling with tdTomato and Tuj 1 counterstaining. $B, C$, Sample images of sparsely labeled neurons from cochlear cultures treated with $23 \mathrm{~nm}$ Control- $\mathrm{Fc}(\boldsymbol{B})$ or Sema $5 \mathrm{~B}-\mathrm{Fc}(\boldsymbol{C})$ stained with anti-dsRed antibodies (to label tdTomato) in red and $3 \mathrm{D}$ Imaris reconstructions shown in white. $\boldsymbol{D}$, Outlines of sample neurons cultured in Control-Fc or Sema5B-Fc illustrating differences in branching morphology. $\boldsymbol{E}$, Stick models of sample neurons depict how morphology affects calculated parameters of branch number, average branch depth, and average branch length. $\boldsymbol{F}-\boldsymbol{H}$, Sema5B-Fc-treated SGNs at P0 show significantly fewer branches ( $\boldsymbol{F}$ ), decreased average branch depth $(\boldsymbol{G})$, and increased average branch length $(\boldsymbol{H})$, compared with those treated with Control-Fc [Student's t test; $\mathrm{mean} \pm \mathrm{SEM}^{*}{ }^{*} p \leq 0.05,{ }^{* *} p \leq 0.01$, ${ }^{* * *} p \leq 0.001$; P0 samples; $n=77$ Control-Fc SGNs (4 cochleae); 84 Sema5B-Fc SGNs (4 cochleae)]. I, Sema5B-Fc-treated P0 SGNs have similar total branch lengths compared with those treated with Control-Fc. $J$, PO SGNs from both conditions show an inverse relationship between branch number and average branch length, but have significantly different slopes (ANCOVA; $\left.{ }^{*} p=0.0284\right)$. Scale bar (in $A$ ): $A, \sim 40 \mu \mathrm{m} ; \boldsymbol{B}, C, 12 \mu \mathrm{m}$. n.s. $=$ not significant.

stituents of the SGN peripheral axons (i.e., membranes and cytosolic content), and did not necessarily eliminate them. When we plotted SGN terminal branch number against average branch length (Fig. $3 J$ ), we found these parameters correlated inversely in both treatment groups (Control-Fc slope $=-0.035$; Sema5BFc slope $=-0.063$ ) and the difference between groups was sta- tistically significant ( $p=0.028$ ). So, SGNs with fewer numbers of branches tend to have longer individual branches (and vice versa), and the addition of exogenous Sema5B exaggerated this effect. These data suggest that Sema5B does not cause an overall SGN retraction response, but that Sema5B may alter how the SGN branches are configured during the refinement process. 

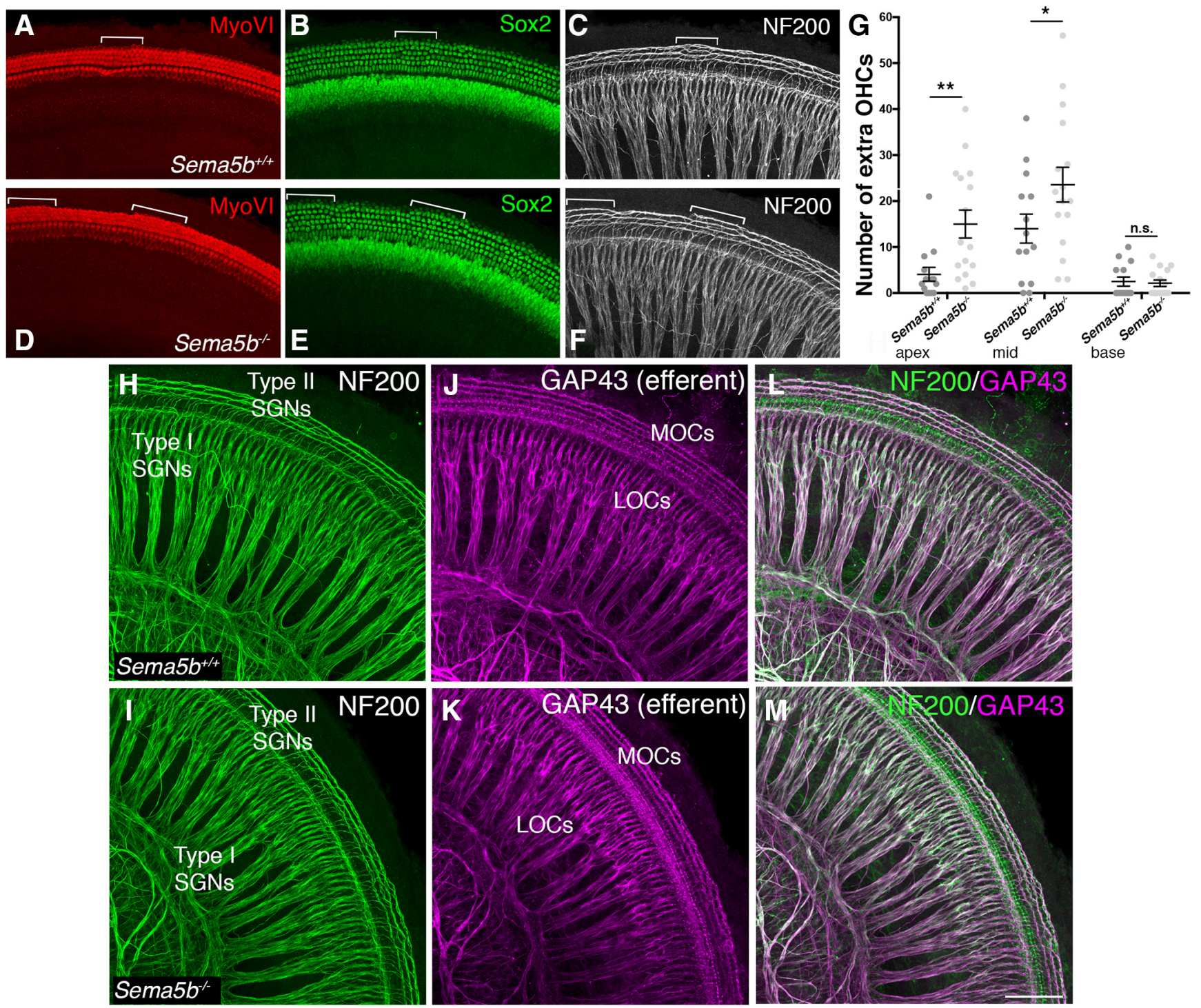

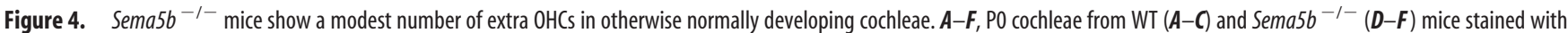
anti-MyoVI to label hair cells, anti-Sox2 to label supporting cells, and anti-NF200 to label SGNs. Extra OHCs, identified by brackets, are observed in both WT (A) and Sema5b ${ }^{-1-}(\boldsymbol{D})$ cochleae. Extra $^{-1}$ OHCs are innervated by type IISGNs $(\boldsymbol{C}, \boldsymbol{F}) . \mathbf{G}$, Sema $5 b^{-1-}$ cochleae show increased numbers of extra OHCs in the apical and middle turns compared with WT controls (Student's $t$ test; mean \pm SEM; ${ }^{*} p \leq 0.05,{ }^{* *} p \leq 0.01 ; n=14$ cochleae WT; 16 cochleae Sema5B ${ }^{-1-}$ ). $\boldsymbol{H}-\mathbf{M}, \mathrm{SGN}$ and olivocochlear efferent (labeled by Gap43) distributions are overall similar in both WT and Sema5b ${ }^{-1-}$ cochleae. LOC, lateral olivocochlear efferent neuron; MOC, medial olivocochlear efferent neuron. Scale bar (in $\boldsymbol{M}$ ): $\boldsymbol{A}-\boldsymbol{F}, \boldsymbol{H}-\boldsymbol{M}, \sim 100 \mu \mathrm{m}$. n.s. $=$ not significant.

\section{Sema $5 b^{-1-}$ mice show a modest number of extra OHCs in} otherwise normally formed cochleae

To begin to investigate Sema5B function in vivo, we first examined cochleae from a Sema $5 b^{-1-}$ mouse line, which had previously been characterized in the context of neuron segregation in the retina (Matsuoka et al., 2011). Compared with littermate controls at P0-P1, Sema5b $b^{-1-}$ cochleae showed an overall normal distribution of hair cells and supporting cells (Fig. 4; MyoVI and Sox2 staining, respectively). The mammalian cochlea typically has a single row of IHCs and three rows of OHCs, but variability in this pattern exists in certain species (Manley, 2012). In the wild-type (WT) mouse cochlea, it is typical to see small patches of extra OHCs (a small fourth row), especially near the middle of the cochlea, and these small patches are usually accompanied by supporting cells and type II SGNs (Fig. $4 A-C$, bracketed region). Notably, the Sema $5 b^{-1-}$ cochleae showed elevated numbers of these small patches of fourth row OHCs, which also had associated supporting cells and type II SGNs (Fig. 4D-F). The increase in extra OHCs in the Sema5b $b^{-1-}$ cochleae was statistically significant compared with controls but quite small: mutants averaged 40 extra OHCs compared with 21 in controls and this defect was absent from the base (Fig. $4 G$ ). Sema $5 b^{-1-}$ cochleae also showed no apparent defects in cochlear length, and phalloidin staining of the stereocilia bundles showed no changes in hair cell orientation (data not shown). Aside from the small increase in extra $\mathrm{OHC}$ numbers (19 on average), organ of Corti development in mice lacking Sema5b was normal.

\section{Sema $5 b$ is required for normal SGN branching}

We next assessed general patterns of cochlear innervation in Sema $5 b^{-1-}$ and littermate control cochleae using anti-NF200 antibodies, which preferentially label SGNs (Harley et al., 2018) and anti-GAP43 antibodies, which preferentially label efferent fibers (Simmons et al., 1996). Previously, we showed high-magnification micrographs demonstrating that NF200 and GAP43 antibodies clearly delineate these different classes of fibers in the 
early postnatal cochlea (Harley et al., 2018). As shown in Figure 4, $H$ and $I$, the SGN radial bundles and fibers contacting the IHCs (type I SGNs) were similar in the Sema5b $b^{-1-}$ and control cochleae. Type II SGNs in Sema $5 b^{-1-}$ cochleae also appeared to be unaffected and there was no change in the number of NF200labeled fibers extending into the OHC region (Fig. 4H,I). We also found no visible defects in patterning of medial or lateral olivocochlear efferents in the Sema5b $b^{-1-}$ cochleae (Fig. 4J, K, MOCs and LOCs), and the SGNs and efferents showed normal patterns of spatial overlap $(L, M)$. Given there were no gross defects in SGN development or cochlear innervation, we next took a high-resolution view of individual SGNs by evaluating cochleae from Sema5 $b^{-1-}$ mice and littermate controls that also carried the $N^{\text {CreERT2 }} ; R^{\text {Tom }}$ alleles. Figure $5, A$ and $B$, shows examples of individual neurons in each genotype generated from whole-mount confocal images. Morphologies of individual neurons were analyzed using Imaris $3 \mathrm{D}$ reconstructions (Fig. 5A, $B$, white fill), similar to the analyses of the cultured cochleae in Figure 3. Using this approach, we could easily see that the more refined peripheral processes typically observed at $\mathrm{P} 0$ were not as prevalent in SGNs lacking Sema5b (Fig. 5C). After morphometric quantifications, we found that SGNs lacking Sema5b had more numerous terminal branches compared with controls, along with increased branch depth values (Fig. $5 D, E$ ). Interestingly, loss of Sema $5 b$ also yielded SGNs with reduced branch lengths (Fig. 5F). Notably, these effects in the Sema5b knock-out tissue are the opposite from what was observed in the gain-of-function experiments with Sema5B-Fc where SGNs showed reduced terminal branch numbers, and increased branch lengths. If Sema5B normally acted as a potent repulsive factor, we might expect Sema $5 b$ mutants to show more numerous and longer SGN terminal branches, but this was clearly not the case. Loss of Sema $5 b$ led to a significant increase in the accumulated sums of the terminal branch lengths per neuron (Fig. $5 G$ ), probably because of the increased numbers of branches. As we did with the in vitro studies, we plotted SGN terminal branch number against branch length (Fig. 5H). Regardless of genotype, these parameters correlated inversely (WT slope $=-0.381 ; \mathrm{Sema}_{5} b^{-1-}$ slope $=$ $-0.083)$ and the difference between the groups was statistically significant $(p<0.0001)$. So, SGNs with relatively few branches tend to have longer individual branches (and vice versa), and the absence of Sema $5 b$ subdues this effect. Overall, these data support the model that Sema5B normally controls how SGN branches are configured during development without causing axonal repulsion per se.

We note here that it is likely not the case that SGNs in the Sema $5 b^{-1-}$ cochleae experience developmental delay. Our evidence for this comes from a separate, unpublished study using Sox $2^{\text {CreERT2 }}$ sparse labeling in WT cochleae at P0: more mature SGNs at the cochlear base show significantly fewer terminal branches compared with less mature SGNs at the cochlear apex (Student's $t$ test; $p=0.021$; mean \pm SEM; apex: $18.347 \pm 0.717$, $n=98$; base: $15.636 \pm 0.833, n=93$ ). Importantly, SGNs from the more mature base did not show increased branch lengths compared with SGNs at the apex [Student's $t$ test; $p=0.270$ (n.s.); mean \pm SEM; apex: $3.527 \pm 0.105, n=98$; base: $3.714 \pm$ $0.132, n=93]$. So, over the normal course of development, type I SGN peripheral axons shed their terminal branches without greatly increasing the length of each individual branch.

We next asked whether the changes in SGN branching patterns found in the Sema5b $b^{-1-}$ cochleae would confer auditory dysfunction. To address this, four Sema5b ${ }^{-1-}$ mice (2 males, 2 females) and four WT littermate controls ( 2 males, 2 females) were tested for ABR thresholds at P40. ABR thresholds at frequencies between 8 and $32 \mathrm{kHz}$ were measured (Fig. $5 \mathrm{I}$ ) and, when data were analyzed for change in hearing thresholds or wave I amplitude (Fig. 5I; and data not shown), no significant differences were detected. This suggests the loss of Sema5b does not cause significant hearing impairment.

\section{PlexinA1 is required in developing SGNs for normal branching patterns}

We next took a genetic loss-of-function approach to ask whether PlexinA1, a known Sema5B receptor (Matsuoka et al., 2011), might mediate the branch limiting effects of Sema5B. PlexinA1 is expressed by both SGNs and OHCs (Fig. 2), so we selectively removed Plxnal in SGNs using Bhlhb5 ${ }^{\text {Cre }}$ (Ross et al., 2010) and a Plxnal conditional knock-out allele (Yoshida et al., 2006). $B h l h b 5^{\text {Cre }}$ is expressed by SGNs in the cochlea (Appler et al., 2013) and was shown previously to generate SGN conditional knock-outs (Ghimire et al., 2018). Compared with SGNs from controls, which showed discrete PlexinAl protein on the membranes of their somata (Fig. $6 B, C$, yellow arrowhead), SGNs from $B h l h b 5^{\mathrm{Cre}} ;$ Plxna $1^{\mathrm{f} / \mathrm{fl}}$ lacked this staining (Fig. $6 D, E$ ), suggesting efficient Plxnal removal. We note here that we also attempted to remove Plxna3 using an uncharacterized Plxna3 conditional line and multiple Cre lines, but PlexinA3 protein levels were never different from controls, suggesting inadequate recombination or other issues. We next tested the hypothesis that Plxnal conditional knock-out (CKO) mice would show increased SGN branch numbers at P0, similar to what was observed in the Sema5b $b^{-1-}$ cochleae. In this case, we were unable to analyze individual SGNs to count terminal branches (all SGNs express Cre; Fig. 6A), so we used NF200 immunostaining to carefully examine the branching profiles of the type I SGNs around the IHCs (Fig. $6 F, G$ ). In many cases, excessive branching was indeed visible in the Plxna1 CKO cochleae, and this was particularly conspicuous around the region of the pillar cells (Fig. 6G, yellow arrowhead) where small SGN branches are usually limited. To quantify this effect, we first converted each confocal $z$-stack to a binary image with equivalent fluorescence intensity thresholding (see Materials and Methods; Fig. $6 H, I$ ). We then captured a ROI around the IHC region (Fig. $6 \mathrm{H}, \mathrm{I}$, dashed green lines) at the base, middle, and apex regions for each cochlea, and calculated the relative area occupied by the SGN processes. We rationalized that increased numbers of branches would occupy more visible area. Overall, for each region of the cochlea, the Plxna1 CKOs showed an increase in the area occupied (Fig. $6 J$ ), but only in the middle turn of the cochleae were the data statistically significant $(p=0.02)$. Although these effects were limited to only one region of the cochlea, the loss of Plxna1 in SGNs led to increased SGN branching, which was similar to the increased numbers of terminal branches found in the Sema $5 b^{-1-}$ cochleae. These data suggest that PlexinAl on the SGNs may respond to Sema5B from the hair cells to limit SGN branching during hair cell innervation.

\section{Discussion}

Neuronal morphology and connectivity patterns are dictated by a multitude of intracellular and intercellular cues. In the cochlea, type I SGNs develop single synaptic connections with IHCs, following a refinement process involving the elimination of extra axonal protrusions, or "debranching" (Fig. 1). Our findings here suggest Sema5B, and its receptor PlexinA1, mediate SGN debranching by limiting numbers of terminal branches over time. Mechanistically, it is possible that Sema5B signaling induces either a local branch retraction event or, alternately, a local branch 

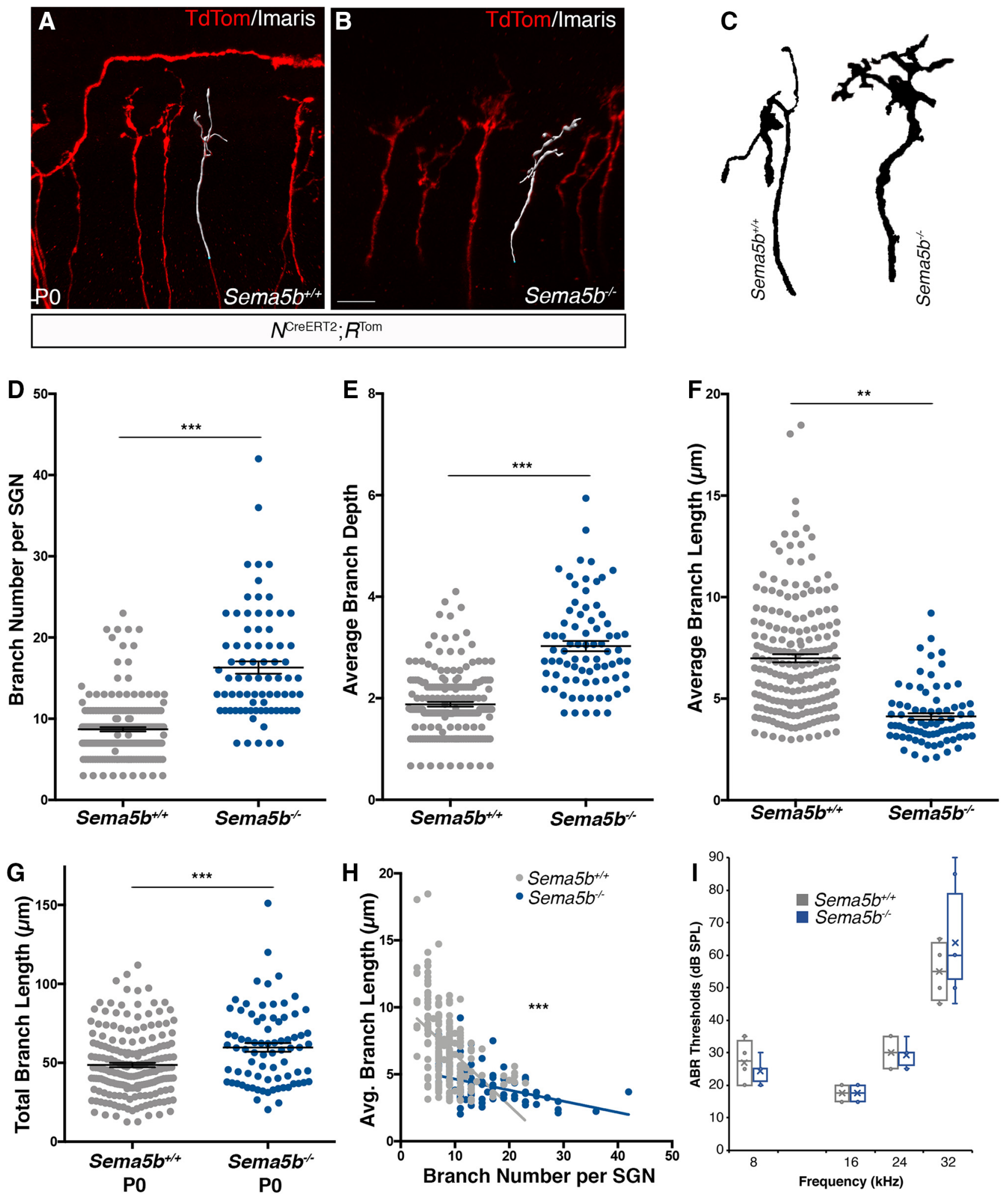

Figure 5. Sparse labeled SGNs show innervation defects in Sema $5 b^{-1-}$ mice. $\boldsymbol{A}, \boldsymbol{B}$, Sample images of sparsely labeled $N^{\text {CreeRT2}}, R^{\text {Tom }}$ neurons from WT $(\boldsymbol{A})$ or Sema5b $b^{-1-}(\boldsymbol{B})$ mice stained with dsRed and 3D reconstructions shown in white. $\boldsymbol{C}$, Outlines of sample neurons from WT or Sema5b $b^{-1-}$ mice show differences in branching morphology. D-F, At P0, SGNs from Sema5 $b^{-1-}$ mice show significantly more branches $(\boldsymbol{D})$, increased average branch depth $(\boldsymbol{E})$, and decreased average branch length $(\boldsymbol{F})$, compared with WT controls [Student's $t$ test; mean \pm SEM; ${ }^{* *} p \leq 0.01$, ${ }_{* * *}^{*} \leq 0.001 ; n=200$ WT SGNs (5 cochleae); 78 Sema5B ${ }^{-1-}$ SGNs (4 cochleae)]. G, SGNs from Sema5b ${ }^{-1-}$ have significantly greater total branch lengths compared with those from WT controls. $\boldsymbol{H}$, SGNs from both WT and Sema5b $b^{-1-}$ mice both show inverse correlations between branch number and average branch length, but have significantly different slopes [ANCOVA; *** $p<0.0001$; $n=200$ WT SGNs (5 cochleae); 78 Sema5B ${ }^{-1-}$ SGNs (4 cochleae)]. I, Sema5b ${ }^{-1-}$ mice show no apparent hearing deficit, as measured by ABR, compared with WT controls. Scale bar (in $\boldsymbol{B}$ ): $\boldsymbol{A}, \boldsymbol{B}$, $\sim 12 \mu \mathrm{m}$. 

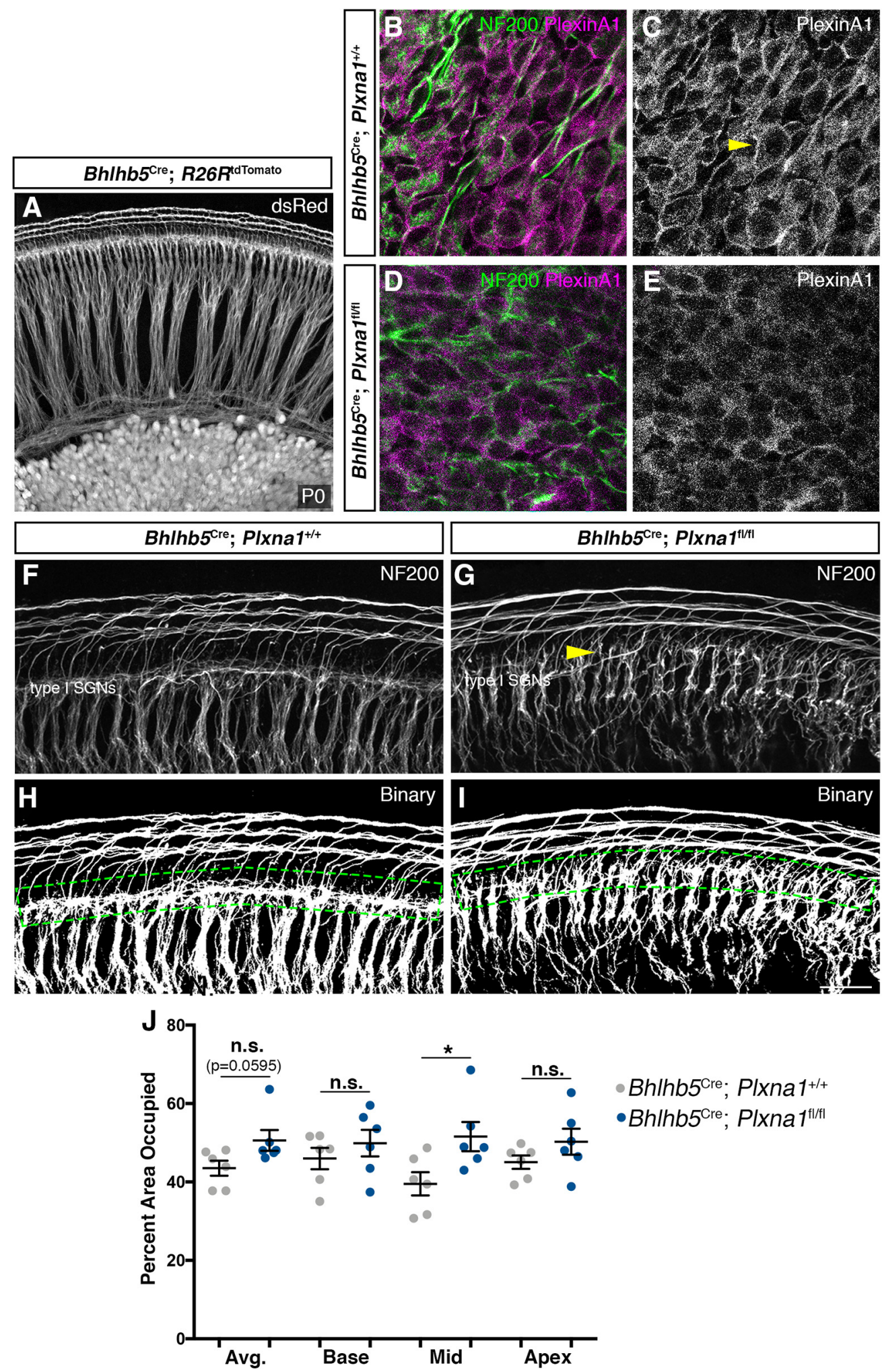

Figure 6. SGNs occupy increased area in Plxna1 mutants. $\boldsymbol{A}$, A whole-mount view from a $B h l h b^{\text {Cree, }}, R 26 R^{\text {tdTomato }}$ cochlea demonstrates the high penetrance of the $B h l h b^{\text {Cre }}$ in $S G N s$. $\boldsymbol{B}-\boldsymbol{E}$, Compared with Bhlhb ${ }^{\text {Cre, }}$;lxna ${ }^{+/+}$controls $(\boldsymbol{B}, \boldsymbol{C})$, levels of PlexinA1 are clearly reduced in SGN membranes (C, arrowhead) of Bhlhb ${ }^{\text {Cree }}$; Plxna ${ }^{\text {fl/fl }}$ mice $(\boldsymbol{D}, \boldsymbol{E}) . \boldsymbol{F}, \boldsymbol{G}$, Sample images of $B h / h b^{\mathrm{Cre}}$; Plxna ${ }^{+/+}(\boldsymbol{F})$ and Bhlhb ${ }^{\mathrm{Cre}} ;$ Plxna $1^{\mathrm{fl} / \mathrm{fl}}(\boldsymbol{G})$ SGNs stained with NF200 in the sensory domain. $\boldsymbol{H}, \boldsymbol{I}$, Resulting images of binary processing of $\boldsymbol{F}$ and $\boldsymbol{G}$ and drawing ROls, shown by green dashed lines. $J$, At the middle turn of the cochlea, SGNs from Bhlhb ${ }^{\text {Cre }} ;$ Plxna $1^{\mathrm{fl} / \mathrm{fl}}$ mice occupy significantly more area than those in Bhlhb ${ }^{\text {Cre; }} ;$ Plxna ${ }^{+/+}$mice (Student's $t$ test; mean $\pm S E M ;{ }^{*} p=0.0307 ; n=$ 6 cochleae per genotype). Scale bar (in $\boldsymbol{I}$ ): $\boldsymbol{A}, \sim 100 \mu \mathrm{m} ; \boldsymbol{B}-\boldsymbol{E}, 16 \mu \mathrm{m}, \boldsymbol{F}-\boldsymbol{I}, 30 \mu \mathrm{m}$. n.s. $=$ not significant. 

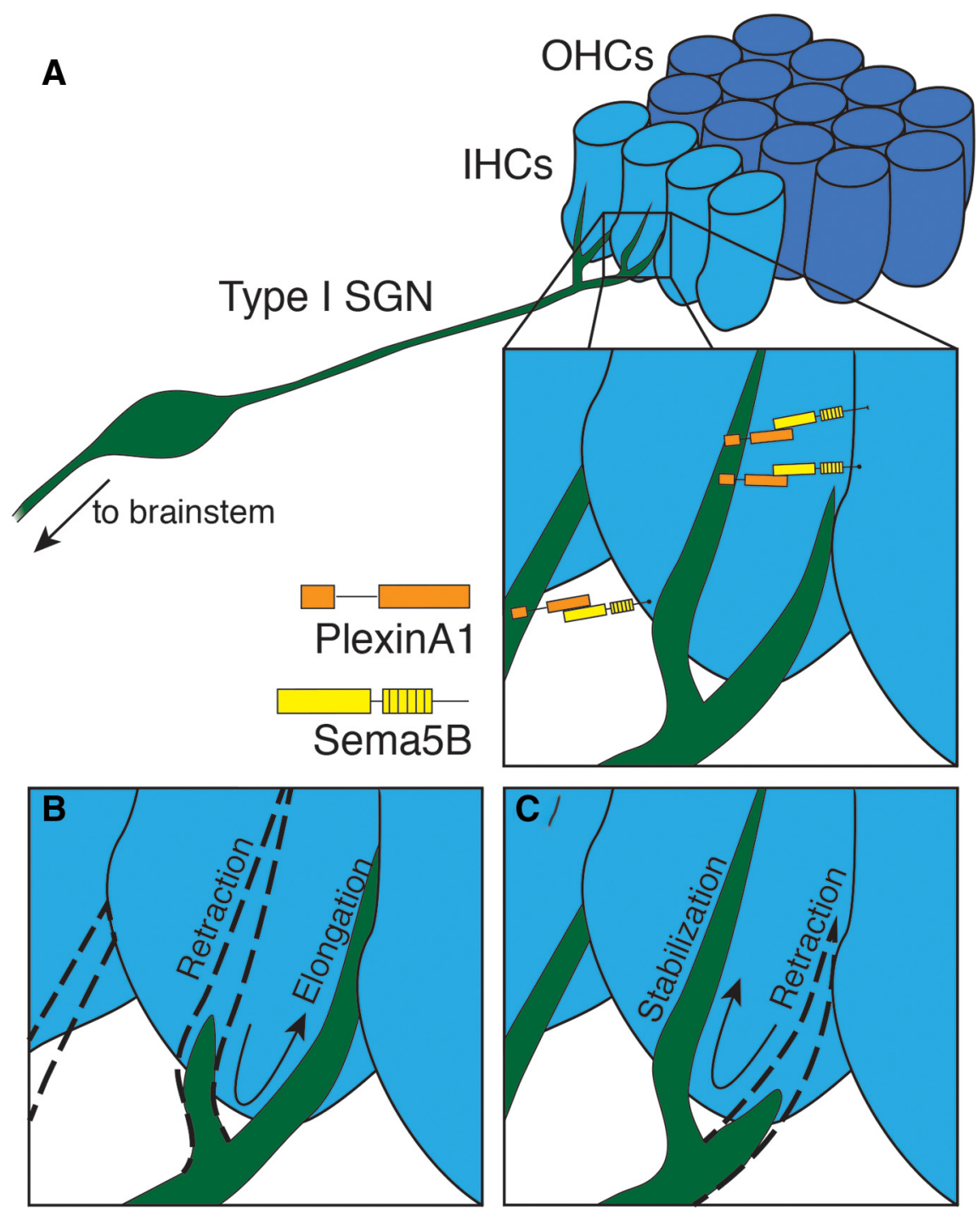

D

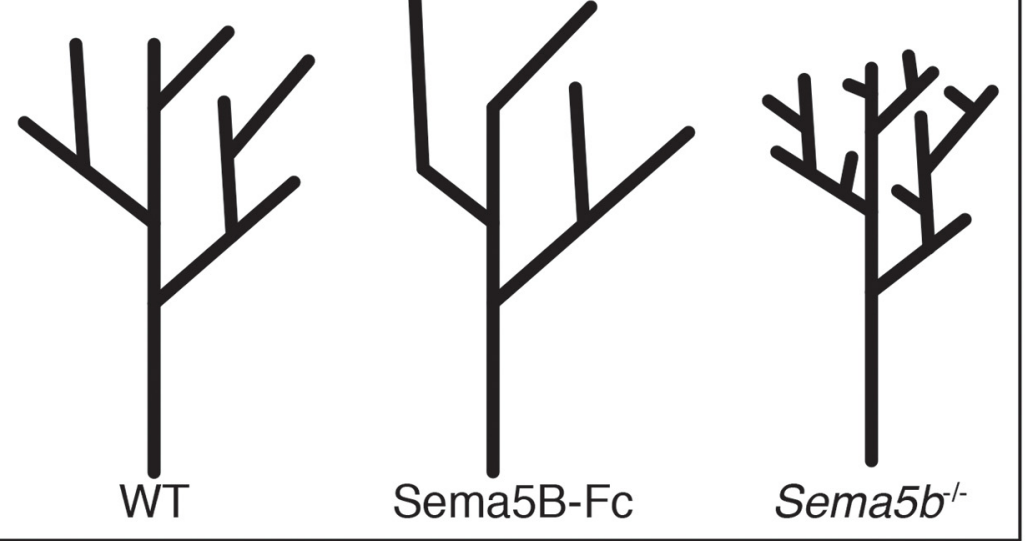

Figure 7. Proposed models of how Sema5B/PlexinA1 signaling could regulate debranching of type ISGNs. A, Transmembrane Sema $5 B$ on hair cells signal through PlexinA1 receptors on developing type ISGNs during late embryonic and early postnatal stages. $B$, Sema5B signaling may cause localized branch retraction and redistribution of cytoskeletal content to other, existing branches. C, Sema5B signaling may locally stabilize select branches while another mechanism causes retraction in other branches. D, Stick models demonstrating morphologic trends of SGNs in the various Sema5B signaling environments discussed in this study.

stabilization event. In the context of Sema5B signaling, changes in branch length appear to compensate for changes in branch number: Sema5B-Fc gain-of-function experiments led to fewer, but longer terminal branches (Figs. 3, 7), whereas loss of Sema5b led to more numerous, but shorter terminal branches (Figs. 5, 7). We predict that Sema5B regulates SGN debranching to ensure some of the SGNs remain in contact with hair cells; the elimination of all branches would prevent SGN-hair cell contacts and subsequent synapse formation. One possibility is that Sema5Bmediated retraction events subsequently lead to the elongation of neighboring branches because cytoskeletal or other cytosolic components are redistributed (Fig. $7 B$, arrow). Alternatively, Sema5B may stabilize select branches while others retract in response to nearby repulsive cues (Fig. $7 C$, arrow). The actions of either mechanism could underlie our observations reported here. As shown in Figures 1 and 2, Sema5B is expressed transiently in hair cells and is eliminated by P5. Given that SGN refinement events occur over an extended period (Huang et al., 2007; Liberman and Liberman, 2016; Coate et al., 2019), it is our expectation that the actions of Sema5B/PlexinA1 signaling represent only one component of this process. Other later events, such as thyroid hormone-mediated synaptic pruning signaling (Sendin et al., 2007; Sundaresan et al., 2016), likely also contribute to the entire refinement process.

Our analyses of PlexinA1 and PlexinA3 expression are based largely on the signaling relationship between Sema5B and PlexinAs described in the mouse retina (Matsuoka et al., 2011). Observing PlexinA1 and PlexinA3 expression in SGNs at the same developmental period as Sema5B expression in HCs was the first strong indicator that Sema5B signaling may be influencing SGN morphology in the cochlea. As shown in Figure 6, loss of Plxna1 in SGNs led to increased branching, similar to what we found with the Sema $5 b$ mutants, suggesting PlexinA 1 and Sema5B are in the same pathway. Curiously, though, Plxnal loss in SGNs only led to significant differences in branch patterns in the middle turn of the cochlea. SGNs along the entire length of the cochlea appear to express Plxnal mRNA at uniform levels (Fig. 1), so regional differences in sensitivity to Plxnal loss were not expected. One possibility is that PlexinA3 acts redundantly to PlexinA 1 as in the retina (Matsuoka et al., 2011), but only partially redundant in the middle turn of the cochlea. The SGNs also express mRNAs for Plexins A4 (Suto et al., 2003), B1, B2, C1, and D1 (Lu et al., 2011), and these may also act partially redundant to PlexinAl. In future studies, it will be important to understand the extent to which additional Plexins and other factors also contribute to SGN refinement. 
The loss of Sema5b and the described changes in SGN refinement did not yield significant increases in ABR thresholds (Fig. 5) at the one age examined (P40). It is possible that the ABR test lacks the sensitivity to detect differences in SGN firing characteristics present in the Sema $5 b^{-1-}$ null mice, or that any defects in innervation were compensated for by synaptic pruning events ( $\sim \mathrm{P} 8-\mathrm{P} 10)$ that follow the period of Sema5B expression, which ends at $\sim$ P5 (Fig. 1). Previously, we documented how cochlear morphology and ribbon synapse distribution were able to "recover" in $\mathrm{NrCAM}^{-1-}$ mice (Harley et al., 2018) and a similar kind of recovery in the Sema5 $b^{-1-}$ mice may lead to limited changes in adult hearing thresholds. Our hearing assessment was underpowered to detect very subtle differences in hearing thresholds or wave I amplitude, and it is possible that slight differences may be detected with a larger cohort of mice in which males and females are analyzed separately (Milon et al., 2018). It is also possible that loss of Sema5b could somehow lead to progressive hearing loss, which was previously observed in Nrp1 conditional knock-outs (Salehi et al., 2017). Future studies should also examine the influence of Sema5B and SGN branch refinement on the multitude of later events important in SGN development, which extends through P30 in mouse (Liberman and Liberman, 2016; Wu et al., 2016). This includes possible influences on SGN specification (Petitpré et al., 2018; Shrestha et al., 2018; Sun et al., 2018), ribbon synapse formation and pruning (Bulankina and Moser, 2012; Yu and Goodrich, 2014), spontaneous activity (Zhang-Hooks et al., 2016; Babola et al., 2018), and the maturation of SGN firing characteristics (Wu et al., 2016).

Several studies have identified Semaphorin signaling as a regulator of neuronal branching, so there is precedent for the observations we report here. However, much of this work has been limited primarily to class-3 (secreted) Semaphorins, namely Sema3A and Sema3F, in the CNS. For example, axons from visual cortex neurons in the corticospinal tract require PlexinA3 to undergo stereotyped pruning processes and Sema3F is thought to mediate this effect (Low et al., 2008). Similarly, in hippocampal infrapyramidal bundle neurons, Plxna3 mutants do not exhibit typical pruning events and the addition of Sema3A resulted in increased branch retraction in CA1 neurons in vitro (Bagri et al., 2003). Our findings suggest a novel debranching role for Sema5B signaling that is distinct from, yet likely related to, previous characterizations of Sema5B as a traditional chemorepellent (Liu et al., 2014). Our description of the observed effects as a reorganization, rather than an elimination, of branches was made possible only by genetic sparse labeling, which permitted the careful analysis of hundreds of individual neurons. From these studies, we found an inverse correlation between numbers of terminal branches and the branch length both in vitro (Sema5B gain-offunction studies; Fig. 3) and in vivo (Sema5B loss-of-function studies). In our culture experiments, we found no difference in accumulated SGN branch length when cochleae were exposed to Sema5B-Fc, suggesting that the "eliminated" terminal branches may be redistributed to other regions of the peripheral axon. Curiously though, Sema5B-Fc treatment did not lead to significant SGN morphological changes when applied to E18.5 cochleae, even though both Sema5B and PlexinA1 are expressed at this stage. Possibly, increases in SGN activity from E18 to P0, or other unidentified regulatory mechanisms related to PlexinA1 signaling account for this difference.

Interestingly, the Sema5b $b^{-1-}$ cochleae also showed a small increase in numbers of extra OHCs (Fig. 4), so it is possible that Sema5B/Plexin interactions also regulate hair cell numbers. One possibility is that Sema5B/Plexin signaling somehow interacts with Notch signaling (Kiernan, 2013) or other signaling cascades that regulate hair cell numbers. In these studies, we used $G f i 1^{\mathrm{Cre}}$ to remove Plxnal from hair cells (Fig. 2), but no additional OHCs resulted. In these experiments, it is possible that the recombination effects of Gfil ${ }^{\text {Cre }}$ occurred too early in development compared with the time period when PlexinA1 is important for organ of Corti patterning. Or, it is possible that Sema5B interacts with other Plexins in this process, or functions somehow in defining boundaries between hair cells and adjacent outer sulcus cells, similar to BMP signaling (Ohyama et al., 2010). In future studies, the contribution of Sema5B/Plexin signaling to organ of Corti patterning should be more fully addressed.

The results presented here on Sema5B and type I SGN branch refinement support a model whereby different Semaphorins show differing patterns of expression and serve distinct functions in cochlear innervation. Sema3A is expressed by supporting cells on either side of the organ of Corti, and by SGNs, and mice that lack Nrpl in the cochlea show defects in cochlear innervation and SGN survival (Salehi et al., 2017). Sema3F is expressed by hair and supporting cells of the lateral (abneural) region of the organ of Corti and is required for IHC targeting by type I SGNs (Coate et al., 2015). Although functional data are lacking, Sema3C is expressed by SGNs, greater epithelial ridge cells, and by hair cells at very low levels, and Sema3D is expressed by greater epithelial ridge cells and by cells of the stria vascularis (Coate et al., 2015). Sema5A is expressed by otic mesenchyme cells (Allen Institute for Brain Science, 2015), but Sema5A function in cochlear development is unknown. With respect to this report, what cytosolic signaling mechanisms might facilitate Sema5B-mediated branch refinement? Broadly, Plexins contain intracellular Rho binding domains that bind Rho family GTPases, as well as GAP domains that can regulate Ras and Rap small GTPases to control cytoskeletal assembly (Alto and Terman, 2017). In the future, it will be important to understand how each of the Semaphorins noted above control different aspects of SGN maturity and cochlear innervation, and it will be important to understand any variations in cytosolic signaling responses in SGNs that may result from exposure to different Semaphorins.

\section{References}

Agmon A, Yang LT, O’Dowd DK, Jones EG (1993) Organized growth of thalamocortical axons from the deep tier of terminations into layer IV of developing mouse barrel cortex. J Neurosci 13:5365-5382

Allen Institute for Brain Science (2015) Allen mouse brain atlas. Available from: http://mouse.brain-map.org.

Alto LT, Terman JR (2017) Semaphorins and their signaling mechanisms. Methods Mol Biol Methods Mol Biol 1493:1-25.

Appler J, Lu C, Druckenbrod NR, Yu WM, Koundakjian EJ, Goodrich LV (2013) Gata3 is a critical regulator of cochlear wiring. J Neurosci 33: 3679-3691.

Babola TA, Li S, Gribizis A, Lee BJ, Issa JB, Wang HC, Crair MC, Bergles DE (2018) Homeostatic control of spontaneous activity in the developing auditory system. Neuron 99:511-524.e5.

Bagri A, Cheng HJ, Yaron A, Pleasure SJ, Tessier-Lavigne M (2003) Stereotyped pruning of long hippocampal axon branches triggered by retraction inducers of the semaphorin family. Cell 113:285-299.

Berglund AM, Ryugo DK (1987) Hair cell innervation by spiral ganglion neurons in the mouse. J Comp Neurol 255:560-570.

Bouvrée K, Brunet I, Del Toro R, Gordon E, Prahst C, Cristofaro B, Mathivet T, Xu Y, Soueid J, Fortuna V, Miura N, Aigrot MS, Maden CH, Ruhrberg C, Thomas JL, Eichmann A (2012) Semaphorin3A, Neuropilin-1, and PlexinA1 are required for lymphatic valve formation. Circ Res 111:437445.

Bulankina AV, Moser T (2012) Neural circuit development in the mammalian cochlea. Physiology 27:100-112.

Coate TM, Spita NA, Zhang KD, Isgrig KT, Kelley MW (2015) Neuropilin- 
2/semaphorin-3F-mediated repulsion promotes inner hair cell innervation by spiral ganglion neurons. eLife 4:e07830.

Coate TM, Scott MK, Gurjar M (2019) Current concepts in cochlear ribbon synapse formation. Synapse 73:e22087.

Defourny J, Poirrier AL, Lallemend F, Mateo Sánchez S, Neef J, Vanderhaeghen P, Soriano E, Peuckert C, Kullander K, Fritzsch B, Nguyen L, Moonen G, Moser T, Malgrange B (2013) Ephrin-A5/EphA4 signalling controls specific afferent targeting to cochlear hair cells. Nat Commun 4:1438.

de Wit J, Verhaagen J (2003) Role of semaphorins in the adult nervous system. Prog Neurobiol 71:249-267.

Driver EC, Pryor SP, Hill P, Turner J, Rüther U, Biesecker LG, Griffith AJ, Kelley MW (2008) Hedgehog signaling regulates sensory cell formation and auditory function in mice and humans. J Neurosci 28:7350-7358.

Druckenbrod NR, Goodrich LV (2015) Sequential retraction segregates SGN processes during target selection in the cochlea. J Neurosci 35:16221-16235.

Fiore R, Püschel AW (2003) The function of semaphorins during nervous system development. Front Biosci 8:s484-499.

Flores EN, Duggan A, Madathany T, Hogan AK, Márquez FG, Kumar G, Seal RP, Edwards RH, Liberman MC, García-Añoveros J (2015) A noncanonical pathway from cochlea to brain signals tissue-damaging noise. Curr Biol 25:606-612.

Ghimire SR, Ratzan EM, Deans MR (2018) A non-autonomous function of the core PCP protein VANGL2 directs peripheral axon turning in the developing cochlea. Development 145:dev159012.

Glasbey CA (1993) An analysis of histogram-based thresholding algorithms. Graph Model Image Process 55:532-537.

Glowatzki E, Fuchs PA (2002) Transmitter release at the hair cell ribbon synapse. Nat Neurosci 5:147-154.

Gu C, Rodriguez ER, Reimert DV, Shu T, Fritzsch B, Richards LJ, Kolodkin AL, Ginty DD (2003) Neuropilin-1 conveys semaphorin and VEGF signaling during neural and cardiovascular development. Dev Cell 5:45-57.

Harley RJ, Murdy JP, Wang Z, Kelly MC, Ropp TF, Park SH, Maness PF, Manis PB, Coate TM (2018) Neuronal cell adhesion molecule ( $\mathrm{NrCAM}$ ) is expressed by sensory cells in the cochlea and is necessary for proper cochlear innervation and sensory domain patterning during development. Dev Dyn 247:934-950.

Huang LC, Barclay M, Lee K, Peter S, Housley GD, Thorne PR, Montgomery JM (2012) Synaptic profiles during neurite extension, refinement and retraction in the developing cochlea. Neural Dev 7:38.

Huang LC, Thorne PR, Housley GD, Montgomery JM (2007) Spatiotemporal definition of neurite outgrowth, refinement and retraction in the developing mouse cochlea. Development 134:2925-2933.

Jongbloets BC, Pasterkamp RJ (2014) Semaphorin signalling during development. Development 141:3292-3297.

Kalil K, Dent EW (2014) Branch management: mechanisms of axon branching in the developing vertebrate CNS. Nat Rev Neurosci 15:7-18

Katayama K, Imai F, Suto F, Yoshida Y (2013) Deletion of Sema3a or plexinA1/plexinA3 causes defects in sensory afferent projections of statoacoustic ganglion neurons. PLoS One 8:e72512.

Kiernan AE (2013) Notch signaling during cell fate determination in the inner ear. Semin Cell Dev Biol 24:470-479.

Koundakjian EJ, Appler JL, Goodrich LV (2007) Auditory neurons make stereotyped wiring decisions before maturation of their targets. J Neurosci 27:14078-14088.

Liberman LD, Liberman MC (2016) Postnatal maturation of auditorynerve heterogeneity, as seen in spatial gradients of synapse morphology in the inner hair cell area. Hear Res 339:12-22.

Liu C, Glowatzki E, Fuchs PA (2015) Unmyelinated type II afferent neurons report cochlear damage. Proc Natl Acad Sci U S A 112:14723-14727.

Liu RQ, Wang W, Legg A, Abramyan J, O'Connor TP (2014) Semaphorin $5 \mathrm{~B}$ is a repellent cue for sensory afferents projecting into the developing spinal cord. Development 141:1940-1949.

Low LK, Liu XB, Faulkner RL, Coble J, Cheng HJ (2008) Plexin signaling selectively regulates the stereotyped pruning of corticospinal axons from visual cortex. Proc Natl Acad Sci U S A 105:8136-8141.

Lu CC, Appler JM, Houseman EA, Goodrich LV (2011) Developmental profiling of spiral ganglion neurons reveals insights into auditory circuit assembly. J Neurosci 31:10903-10918.

Manley GA (2012) Evolutionary paths to mammalian cochleae. J Assoc Res Otolaryngol 13:733-743.
Matern M, Vijayakumar S, Margulies Z, Milon B, Song Y, Elkon R, Zhang X, Jones SM, Hertzano R (2017) GfilCre mice have early onset progressive hearing loss and induce recombination in numerous inner ear non-hair cells. Sci Rep 7:42079.

Matsuoka RL, Chivatakarn O, Badea TC, Samuels IS, Cahill H, Katayama K ichi, Kumar SR, Suto F, Chédotal A, Peachey NS, Nathans J, Yoshida Y, Giger RJ, Kolodkin AL (2011) Class 5 transmembrane semaphorins control selective mammalian retinal lamination and function. Neuron 71:460-473.

Meyer AC, Moser T (2010) Structure and function of cochlear afferent innervation. Curr Opin Otolaryngol Head Neck Surg 18:441-446.

Milon B, Mitra S, Song Y, Margulies Z, Casserly R, Drake V, Mong JA, Depireux DA, Hertzano R (2018) The impact of biological sex on the response to noise and otoprotective therapies against acoustic injury in mice. Biol Sex Differ 9:12.

Murakami Y, Suto F, Shimizu M, Shinoda T, Kameyama T, Fujisawa $\mathrm{H}$ (2001) Differential expression of Plexin-A subfamily members in the mouse nervous system. Dev Dyn 220:246-258.

Nayagam BA, Muniak MA, Ryugo DK (2011) The spiral ganglion: connecting the peripheral and central auditory systems. Hear Res 278:2-20.

O’Connor TP, Cockburn K, Wang W, Tapia L, Currie E, Bamji SX (2009) Semaphorin $5 \mathrm{~B}$ mediates synapse elimination in hippocampal neurons. Neural Dev 4:18.

Ohyama T, Basch ML, Mishina Y, Lyons KM, Segil N, Groves AK (2010) BMP signaling is necessary for patterning the sensory and nonsensory regions of the developing mammalian cochlea. J Neurosci 30: 15044-15051.

Oster SF, Bodeker MO, He F, Sretavan DW (2003) Invariant Sema5A inhibition serves an ensheathing function during optic nerve development. Development 130:775-784

Pasterkamp RJ, Giger RJ (2009) Semaphorin function in neural plasticity and disease. Curr Opin Neurobiol 19:263-274.

Petitpré C, Wu H, Sharma A, Tokarska A, Fontanet P, Wang Y, Helmbacher F, Yackle K, Silberberg G, Hadjab S, Lallemend F (2018) Neuronal heterogeneity and stereotyped connectivity in the auditory afferent system. Nat Commun 9:3691.

Polley DB, Thompson JH, Guo W (2013) Brief hearing loss disrupts binaural integration during two early critical periods of auditory cortex development. Nat Commun 4:2547.

Raphael Y, Altschuler RA (2003) Structure and innervation of the cochlea. Brain Res Bull 60:397-422.

Riccomagno MM, Kolodkin AL (2015) Sculpting neural circuits by axon and dendrite pruning. Annu Rev Cell Dev Biol 31:779-805.

Ross SE, Mardinly AR, McCord AE, Zurawski J, Cohen S, Jung C, Hu L, Mok SI, Shah A, Savner EM, Tolias C, Corfas R, Chen S, Inquimbert P, Xu Y, McInnes RR, Rice FL, Corfas G, Ma Q, Woolf CJ, Greenberg ME (2010) Loss of inhibitory interneurons in the dorsal spinal cord and elevated itch in Bhlhb5 mutant mice. Neuron 65:886-898.

Salehi P, Ge MX, Gundimeda U, Michelle Baum L, Lael Cantu H, Lavinsky J, Tao L, Myint A, Cruz C, Wang J, Nikolakopoulou AM, Abdala C, Kelley MW, Ohyama T, Coate TM, Friedman RA (2017) Role of neuropilin-1/ semaphorin-3A signaling in the functional and morphological integrity of the cochlea. PLoS Genet 13:e1007048.

Schindelin J, Arganda-Carreras I, Frise E, Kaynig V, Longair M, Pietzsch T, Preibisch S, Rueden C, Saalfeld S, Schmid B, Tinevez JY, White DJ, Hartenstein V, Eliceiri K, Tomancak P, Cardona A (2012) Fiji: an open-source platform for biological-image analysis. Nat Methods 9:676-682.

Schuldiner O, Yaron A (2015) Mechanisms of developmental neurite pruning. Cell Mol Life Sci 72:101-119.

Schwarz Q, Waimey KE, Golding M, Takamatsu H, Kumanogoh A, Fujisawa H, Cheng HJ, Ruhrberg C (2008) Plexin A3 and plexin A4 convey semaphorin signals during facial nerve development. Dev Biol 324:1-9.

Sendin G, Bulankina AV, Riedel D, Moser T (2007) Maturation of ribbon synapses in hair cells is driven by thyroid hormone. J Neurosci 27:31633173.

Shepard AR, Scheffel JL, Yu WM (2019) Relationships between neuronal birthdates and tonotopic position in the mouse cochlear nucleus. J Comp Neurol 527:999-1011. 
Shrestha BR, Chia C, Wu L, Kujawa SG, Liberman MC, Goodrich LV (2018) Sensory neuron diversity in the inner ear is shaped by activity. Cell 174:1229-1246.e17.

Simmons DD, Moulding HD, Zee D (1996) Olivocochlear innervation of inner and outer hair cells during postnatal maturation: an immunocytochemical study. Brain Res Dev Brain Res 95:213-226.

Spillane M, Gallo G (2014) Involvement of Rho-family GTPases in axon branching. Small GTPases 5:e27974.

Stanfield BB, O'Leary DD, Fricks C (1982) Selective collateral elimination in early postnatal development restricts cortical distribution of rat pyramidal tract neurones. Nature 298:371-373.

Sun S, Babola T, Pregernig G, So KS, Nguyen M, Su SM, Palermo AT, Bergles DE, Burns JC, Müller U (2018) Hair cell mechanotransduction regulates spontaneous activity and spiral ganglion subtype specification in the auditory system. Cell 174:1247-1263.e15.

Sundaresan S, Kong JH, Fang Q, Salles FT, Wangsawihardja F, Ricci AJ, Mustapha M (2016) Thyroid hormone is required for pruning, functioning and long-term maintenance of afferent inner hair cell synapses. Eur J Neurosci 43:148-161.
Suto F, Murakami Y, Nakamura F, Goshima Y, Fujisawa H (2003) Identification and characterization of a novel mouse plexin, plexin-A4. Mech Dev 120:385-396.

Wu JS, Young ED, Glowatzki E (2016) Maturation of spontaneous firing properties after hearing onset in rat auditory nerve fibers: spontaneous rates, refractoriness, and interfiber correlations. J Neurosci 36:10584-10597.

Yang H, Gan J, Xie X, Deng M, Feng L, Chen X, Gao Z, Gan L (2010) Gfil-cre knock-in mouse line: a tool for inner ear hair cell-specific gene deletion. Genesis 48:400-406.

Yoong LF, Pai YJ, Moore AW (2019) Stages and transitions in dendrite arbor differentiation. Neurosci Res 138:70-78.

Yoshida Y, Han B, Mendelsohn M, Jessell TM (2006) PlexinA1 signaling directs the segregation of proprioceptive sensory axons in the developing spinal cord. Neuron 52:775-788.

Yu WM, Goodrich LV (2014) Morphological and physiological development of auditory synapses. Hear Res 311:3-16.

Zhang-Hooks Y, Agarwal A, Mishina M, Bergles DE (2016) NMDA receptors enhance spontaneous activity and promote neuronal survival in the developing cochlea. Neuron 89:337-350. 